\title{
Inhibition of miR-27a suppresses the inflammatory response via the p38/MAPK pathway in intervertebral disc cells
}

\author{
ZHENGUO CAO $^{1,2}$ and LIANG CHEN ${ }^{1}$ \\ ${ }^{1}$ Department of Orthopaedic Surgery, The First Affiliated Hospital of Soochow University, \\ Suzhou, Jiangsu 215006; ${ }^{2}$ Department of Orthopaedic Surgery, The Second Affiliated \\ Hospital of Xuzhou Medical University, Xuzhou 221006, P.R. China
}

Received July 5, 2016; Accepted April 7, 2017

DOI: $10.3892 / \mathrm{etm} .2017 .5053$

\begin{abstract}
The current study aimed to investigate the role of miR-27a in intervertebral disc degeneration (IDD) and to examine the underlying mechanisms. Quantitative polymerase chain reaction (qPCR) was performed to detect the expression level of miR-27a in the nucleus pulposus (NP) tissues of patients with IDD, and the results revealed an increasing expression of miR-27a in IDD compared with the control. To further investigate the role of miR-27a in IDD, a stable human NP cell line with low miR-27a expression was generated by transfecting cells with a lentiviral antigomiR-27a inhibitor. In addition, a human NP cell inflammation model was established by lipopolysaccharide (LPS; $10 \mu \mathrm{M}$ ) stimulation. The miR-27a expression in NP cells was determined by qPCR, while the expression of its target proteins; p-p38 and nuclear factor $(\mathrm{NF}-\kappa \mathrm{B})$ was measured by western blot analysis. Furthermore, the mRNA and protein expression levels of proinflammatory factors, including interleukin (IL)-1 $\beta$, IL-6 and tumor necrosis factor- $\alpha$ (TNF- $\alpha)$, were also evaluated by qPCR and ELISA, respectively. The current results confirmed that miR-27a was significantly upregulated in IDD. In vitro, downregulation of miR-27a in LPS-stimulated NP cells by transfection with the miR-27a inhibitor resulted in suppression of p-p38 and $\mathrm{NF}-\kappa \mathrm{B}$ expression levels. Furthermore, the production of the proinflammatory factors IL-1 $\beta$, IL- 6 and TNF- $\alpha$ was significantly reduced in LPS-stimulated NP cells with downregulated miR-27a. In conclusion, miR-27a may function as a promoter in IDD development, while inhibition of miR-27a may suppress proinflammatory factors released by intervertebral disc cells by regulating the $\mathrm{p} 38 /$ mitogen-activated protein kinase (MAPK) signaling pathway.
\end{abstract}

Correspondence to: Dr Liang Chen, Department of Orthopaedic Surgery, The First Affiliated Hospital of Soochow University, 188 Shizi Street, Suzhou, Jiangsu 215006, P.R. China

E-mail: chenliang161230@163.com

Key words: microRNA-27a, intervertebral disc degeneration, p38, mitogen-activated protein kinase

\section{Introduction}

As one of the major causes of lower back pain, intervertebral disc degeneration (IDD) remains an important global problem, severely affecting the quality of life and leading to a serious socioeconomic burden (1-3). Multiple risk factors can cause IDD, including genetic predisposition, lifestyle and aging among others $(4,5)$. The incidence of IDD is higher in developing countries, particularly in China. It has been indicated that various cellular events, ranging from matrix synthesis to cytokine expression, are involved in the progression of human IDD (6). Increasing evidence supports the observation that nucleus pulposus (NP) cells, which produce type II collagen, aggrecan and other components of the extracellular matrix (ECM), serve a critical role in maintaining the integrity of intervertebral discs (IVDs) $(7,8)$. In addition, excessive apoptosis of IVD cells and of the components of ECM occurs during the IDD progress. Loss of the proteoglycan (PG) content of IVDs is one of the main features of IDD; thus, as a strong promoter of inflammation, lipopolysaccharide (LPS) can reduce the PG content and cause the occurrence of IDD $(9,10)$. Furthermore, proinflammatory factors, including interleukin-1 $\beta$ (IL-1 $\beta$ ), IL-6 and tumor necrosis factor- $\alpha$ (TNF- $\alpha$ ), also serve important roles in IDD. However, the underlying molecular and cellular mechanisms of IDD remain largely unknown.

MicroRNAs (miRNAs or miRs) are a class of small non-coding RNA molecules with a length of 20-22 nucleotides that serve key roles in post-transcriptional regulation of gene expression $(11,12)$. miRNAs have a central role in the development of cancer, as well as in inflammatory, neurodegenerative and the majority of degenerative disorders $(13,14)$. Recently, multiple studies have demonstrated that miRNAs serve an important role in degenerative disc diseases, such as IDD (15-19). As a multifunctional miRNA, miR-27a is expressed in various tissues, and abnormal expression of miR-27a has been detected in various diseases (20-22). Previously, miR-27a has been reported to be upregulated in human degenerative NP cells when compared with the control NP cells (19); however, the underlying mechanisms remain unknown.

Thus, the present study aimed to verify the expression and to investigate the role of miR-27a in IDD, as well as to examine the underlying mechanisms involved. 


\section{Materials and methods}

Materials. ELISA kits for the detection of IL- $1 \beta$ (Cat no. E-EL-H0149c), IL-6 (Cat no. E-EL-H0102c) and TNF- $\alpha$ (Cat no. E-EL-H0109c) protein levels were obtained from Elabscience Biotechnology Co., Ltd (Wuhan, China). Antibodies against p-p38 (Cat no. 1170), NF-кB (Cat no. 8214) and $\beta$-actin (Cat no. 12620), as well as the secondary antibodies, were supplied by Cell Signaling Technology, Inc. (Danvers, MA, USA). LPS was purchased from Sigma-Aldrich (Merck, Darmstadt, Germany). The miR-27a inhibitor (lentiviral antigomiR-27a) and the cell transfection kit (Cat no. sc-36868) were purchased from Santa Cruz Biotechnology, Inc. (Dallas, TX, USA). All other chemicals and reagents were purchased from Sinopharm Chemical Reagent Co., Ltd. (Shanghai, China).

Specimens. The study was approved by the Human Ethics Committee Review Board at the First Affiliated Hospital of Soochow University (Suzhou, China), and written informed consent was obtained from each patient. In total, 20 patients with degenerative disc disease, presenting with lumbar intervertebral disc herniation (LIDH), a medical condition that is representative of IDD (IDD group) and 20 spinal cord injury patients (control group) were enrolled into the present study. The inclusion and exclusion criteria of the patients were as previously described by Zhao et al (23). The degenerative condition of IDD and IVDs (control patients who were suffering from a lower grade of IDD) was assessed by magnetic resonance imaging (MRI) according to the Pfirrmann's grading system (24). Samples graded as 4 from patients were identified as the IDD group, samples graded 1 from cadaveric donors were classified as the control group. The NP tissues were carefully dissected during the disc excision surgery following a protocol approved by the Institutional Review Board at the First Affiliated Hospital of Soochow University, and then subjected to various methods of analysis, according to the corresponding procedures. Briefly, NP tissues were separated from the annulus using a stereotaxic microscope; herniation tissues and granulation tissues were excluded. Subsequently, as described previously (23), the whole tissue specimens were washed with phosphate-buffered saline $(\mathrm{pH}$ 7.2) and then divided into two sections of equal size. One of the sections was fixed with $4 \%$ paraformaldehyde at $4^{\circ} \mathrm{C}$ for $30 \mathrm{~min}$ and used in subsequent studies, while the remaining section was snap-frozen and stored in liquid nitrogen within $30 \mathrm{~min}$ of removal from the patients, with subsequent storage at $-80^{\circ} \mathrm{C}$, and used in RT-qPCR analysis.

Cell culture. Primary human NP cells (Cat no. \#4800) were purchased from the ScienCell Research Laboratories, Inc. (Carlsbad, CA, USA). Cells were cultured in Dulbecco's modified Eagle's medium (DMEM; Gibco; Thermo Fisher Scientific, Inc., Waltham, MA, USA) supplemented with $10 \%$ fetal bovine serum (Gibco; Thermo Fisher Scientific, Inc.), 1\% L-glutamine (Gibco; Thermo Fisher Scientific, Inc.), $100 \mathrm{mg} / \mathrm{ml}$ streptomycin and $100 \mathrm{U} / \mathrm{ml}$ penicillin were added, and cells were incubated in a $5 \% \mathrm{CO}_{2}$ incubator at $37^{\circ} \mathrm{C}$. Cell culture medium was changed every 2 days and the cells were passaged until they reached $90 \%$ confluence.
Cell transfection and LPS treatment. Human NP cells were plated in a 6 -well plate $\left(5 \times 10^{4}\right.$ cells/well) the day prior to transfection. NP cells were transiently transfected with an miR-27a inhibitor (lentiviral antigomiR-27a; GenScript, Piscataway, NJ, USA) or its negative control (lentiviral vector control; GenScript) with $30 \mu$ l Lipofectamine 2000 transfection reagent (Invitrogen; Thermo Fisher Scientific, Inc.) following the manufacturer's instructions. At $24 \mathrm{~h}$ after the transfection, cells were stimulated with LPS $(10 \mathrm{ng} / \mathrm{ml})$ in serum-free medium for $24 \mathrm{~h}$ at $37^{\circ} \mathrm{C}$ under $5 \% \mathrm{CO}_{2}$. Next, the supernatants were collected by centrifugation $\left(1,000 \mathrm{x} \mathrm{g}\right.$ at $4^{\circ} \mathrm{C}$ for $\left.10 \mathrm{~min}\right) 24 \mathrm{~h}$ after the initiation of treatment for each group. The treatment for each groups is as follows: Con group, cells without any treatment; LPS group, cells treated with LPS; NC/LPS group, cells transfected with negative control and then treated with LPS; 27a/LPS group, cells transfected with miR-27a inhibitor and then treated with LPS. Then cells were harvested for use in subsequent experiments. The NP cells in the control group did not undergo any treatment.

Reverse transcription-quantitative polymerase chain reaction $(R T-q P C R)$. Total RNA from NP tissues or NP cells was isolated using TRIzol reagent (Takara Bio, Inc., Shiga, Japan) and a mirVana PARIS kit (Ambion; Thermo Fisher Scientific, Inc.) following the manufacturer's instructions. The RNA concentration was then quantified using a Nanodrop spectrophotometer (Thermo Fisher Scientific, Inc.) at $260 \mathrm{~nm}$. Next, qPCR was performed for miR-27a expression detection. Briefly, total RNA was reverse transcribed into cDNA using the TaqMan microRNA Reverse Transcription kit (Invitrogen; Thermo Fisher Scientific, Inc.) according to the instructions provided by the manufacturer. Subsequently, qPCR was performed to analyze the synthesized cDNA. The $20 \mu \mathrm{l}$ master mix contained $2 \mu \mathrm{l} 10 \mathrm{x}$ reverse transcription buffer, $1 \mu \mathrm{l}$ dNTPs (100 mM; with dTTP), $3.75 \mu 1$ nuclease-free water, $0.25 \mu \mathrm{l} 2 \mu \mathrm{M}$ forward and reverse primer, $3.5 \mu \mathrm{l}$ distilled $\mathrm{H}_{2} \mathrm{O}$, and $5 \mu \mathrm{l} 1 \mathrm{ng} / \mu \mathrm{l} \mathrm{cDNA}$. The conditions used for amplification were as follows: 40 cycles of denaturation at $95^{\circ} \mathrm{C}$ for $10 \mathrm{sec}$, followed by $60^{\circ} \mathrm{C}$ for $60 \mathrm{sec}$ to allow annealing and extension. The primers used are listed in Table I. The levels of miR-27a were normalized to the level of the GAPDH, which served as an internal control, by using the $2^{-\Delta \Delta \mathrm{Cq}}$ method (25). The experiment was performed three times in triplicate.

Western blot analysis. Western blot analysis was performed using the transfected cell samples using standard methods. Total cellular protein was extracted using radio immunoprecipitation assay buffer (RIPA buffer, Cell Signaling Technology, Danvers, MA, USA). Briefly, cells were washed with cold PBS 3 times for $5 \mathrm{~min}$, then $200 \mu$ l RIPA buffer was added and incubated for $40 \mathrm{~min}$ (on ice), the supernatants were collected by centrifugation $\left(1,000 \mathrm{x}\right.$ at $4^{\circ} \mathrm{C}$ for $15 \mathrm{~min}$ ). A BCA protein assay kit (Thermo Fisher Scientific, Inc.) was used to detect the protein concentration of samples according to the instructions provided by the manufacturer. Next, proteins (25 $\mu \mathrm{g}$ each sample) were resolved by $10 \%$ SDS-PAGE and then transferred to a polyvinylidene fluoride membrane (EMD Millipore, Billerica, MA, USA). Subsequent to blocking in 5\% nonfat dried milk in Tris-buffered saline-Tween 20 for $2 \mathrm{~h}$, the blots were incubated overnight at $4^{\circ} \mathrm{C}$ with a primary antibody against p-p38 (1:1,000), 
Table I. Primer sequences for polymerase chain reaction.

\begin{tabular}{lll}
\hline Gene & \multicolumn{1}{c}{ Forward sequence $\left(5^{\prime}-3^{\prime}\right)$} & Reverse sequence $\left(5^{\prime}-3^{\prime}\right)$ \\
\hline IL-1 $\beta$ & CTGTGACTCGTGGGATGATG & AGGGATTTTGTCGTTGCTTG \\
IL-6 & GTGCTCCTGGTATTGCTGGT & GGCTCCTCGTTTTCCTTCTT \\
TNF- $\alpha$ & CCTGTCTCTTCCTACCCAACC & GCAGGAGTGTCCGTGTCTTC \\
miR-27a & ACAGGCTAGCGCCGCCTAAC & CCTTAAGGCCCAAGATTACG \\
NF-kB & ACACCTCTGCATATAGCGGC & GGTACCCCCAGAGACCTCAT \\
GAPDH & CTTTGGTATCGTGGAAGGACTC & GTAGAGGCAGGGATGATGTTCT
\end{tabular}

IL, interleukin; TNF, tumor necrosis factor; miR, microRNA; NF, nuclear factor; GAPDH, glyceraldehyde 3-phosphate dehydrogenase.
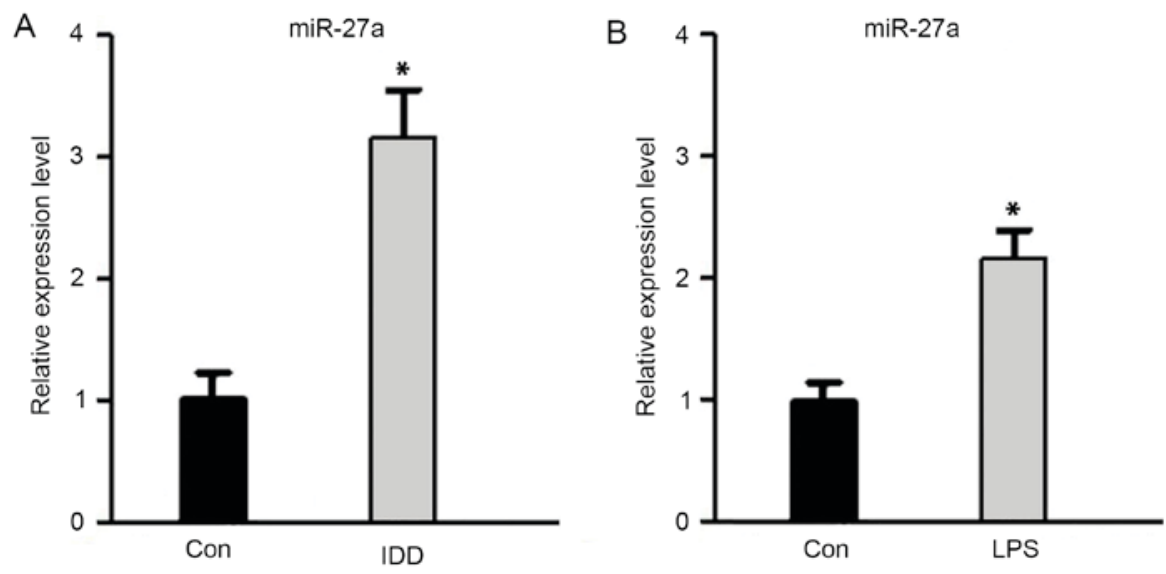

Figure 1. miR-27a expression in (A) tissues of IDD and spinal cord injury patients (control), and (B) NP cells stimulated with LPS to establish an IDD cell model. The relative expression of miR-27a was measured by reverse transcription-quantitative polymerase chain reaction and normalized to GAPDH. miR-27a was upregulated in IDD patients and LPS-stimulated NP cells. "P<0.05 vs. Con group. All results presented as the mean \pm standard deviation of three independent experiments. miR, microRNA; IDD, intervertebral disc degeneration; LPS, lipopolysaccharide; NP, nucleus pulposus; Con, control.

$\mathrm{NF}-\kappa \mathrm{B}(1: 1,000)$ or $\beta$-actin $(1: 2,000)$, and then incubated with a HRP-conjugated secondary antibody (Anti-rabbit IgG; 1:5,000; Cat no. 7074; Cell signaling Technology, Inc., Danvers, MA, USA) at room temperature for $1 \mathrm{~h}$. Protein bands were observed using enhanced chemiluminescence using Super Signal West Pico Chemiluminescent Substrate (Thermo Fisher Scientific, Inc.), and the ImageTool version 3.0 gray-scale scanning software (Microsoft Corporation, Redmond, WA, USA) was applied to quantify the band density. The mean normalized optical density (OD) of the p38 phosphorylation band relative to the OD of the $\beta$-actin band from the same sample was calculated using ImageTool version 3.0 gray-scale scanning software. The expression levels of p38 phosphorylation were expressed as fold changes compared with the control group.

ELISA for IL-1 $\beta, I L-6$ and TNF- $\alpha$ level determination. Cells were stimulated with LPS $(10 \mathrm{ng} / \mathrm{ml})$ in serum-free medium $24 \mathrm{~h}$ after transfection and incubated for a further $24 \mathrm{~h}$ at $37^{\circ} \mathrm{C}$ under $5 \% \mathrm{CO}_{2}$. Then, the supernatants were collected by centrifugation $\left(1,000 \mathrm{x} \mathrm{g}\right.$ at $4^{\circ} \mathrm{C}$ for $\left.10 \mathrm{~min}\right)$. To investigate the expression levels of proinflammatory factors IL-1 $\beta$, IL-6 and TNF- $\alpha$ in the cellular supernatant of the transfected cells following $24 \mathrm{~h}$ of treatment, ELISA was performed, following the manufacturer's instructions of each kit. Each experiment was independently performed three times.
Statistical analysis. Data are displayed as the mean \pm standard deviation. Statistical comparisons between two groups were analyzed with the Student's t-test and between multiple groups with one-way analysis of variance. A difference with a value of $\mathrm{P}<0.05$ was considered as statistically significant.

\section{Results}

Basic patient information. A total of 20 patients with IDD were selected as the IDD group, including 12 males and 8 females, with an age range of 39-69 years and a mean age of $54.9 \pm 7.5$ years. The degenerative IVDs of these patients were the L3-L5 segments, and according to the magnetic resonance imaging (MRI) results, all patients with IDD were classified as grade IV. In addition, 20 patients with spinal cord injury were selected as the control group, including 13 males and 7 females, with an age of 25-56 years and an average age of $42.2 \pm 8.9$ years. The degenerative IVDs of all the control patients (who were suffering from a lower grade of IDD) were the L2-L5 segment, and were classified as grade I based on MRI examination (Table II).

Upregulation of miR-27a expression in IDD. To verify the expression of miR-27a in IDD, RT-qPCR was performed. As shown in Fig. 1, the miR-27a expression level was significantly higher in IDD patients when compared with the control 

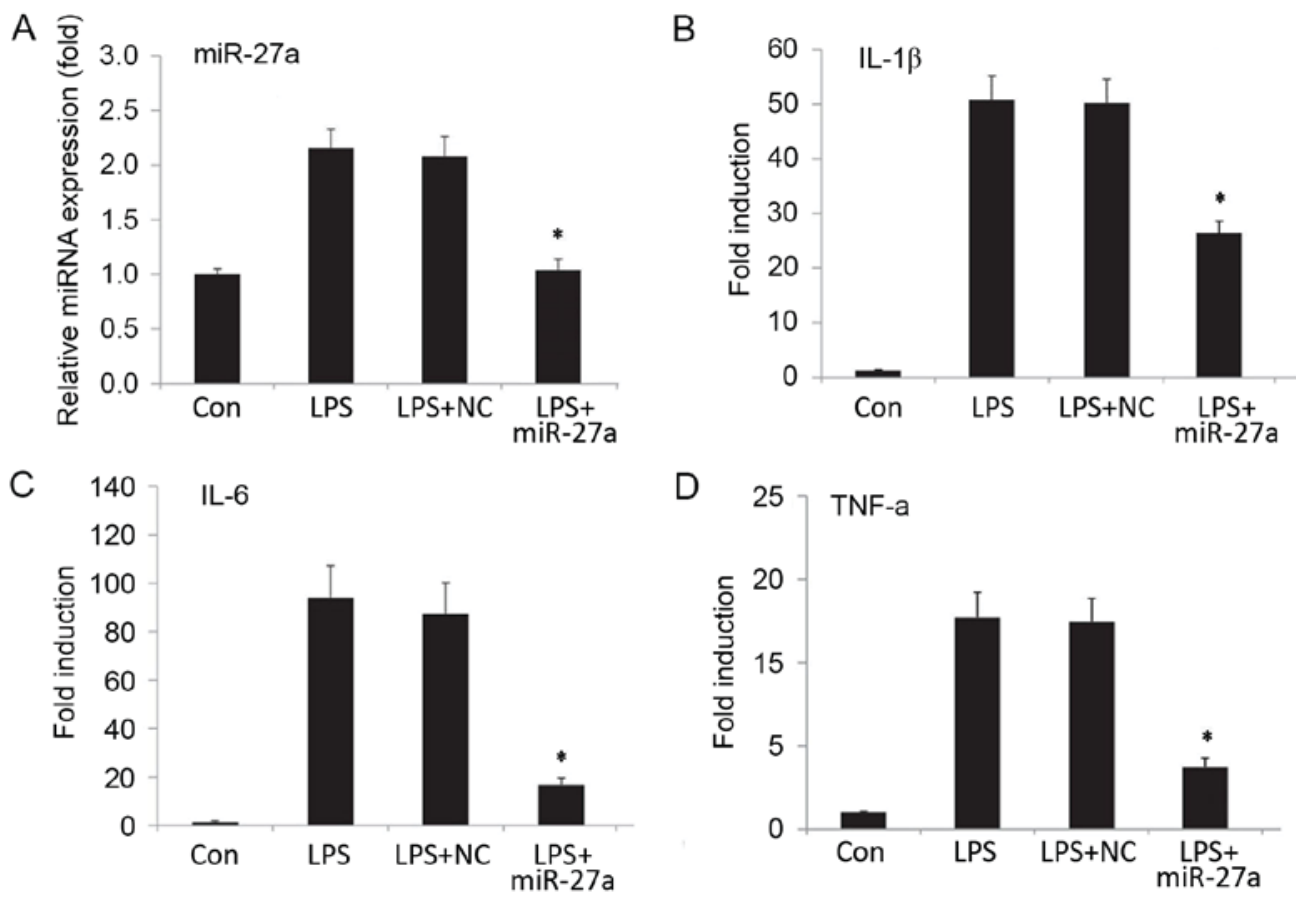

Figure 2. (A) Relative miR-27a expression, and mRNA expression levels of the proinflammatory factors (B) IL-1 $\beta$, (C) IL-6 and (D) TNF- $\alpha$. Human nucleus pulposus cells were transfected with miR-27a mimic or NC (sham transfection without miR-27a, serving as the control group). Next, cells were treated with or without LPS $(10 \mathrm{ng} / \mathrm{ml})$ for $24 \mathrm{~h}$, and expression levels were detected by reverse transcription-quantitative polymerase chain reaction. The relative expression of miRNA/mRNA was normalized to GAPDH. " $\mathrm{P}<0.05$ vs. Con group. All results presented as the mean \pm standard deviation of three independent experiments. miR, microRNA; LPS, lipopolysaccharide; Con, control; NC, negative control vector; IL, interleukin; TNF, tumor necrosis factor; Con, control group, cells without any treatment; LPS group, cells treated with LPS; NC+LPS, cells transfected with negative control and then treated with LPS; LPS+miR-27a, cells transfected with miR-27a inhibitor and then treated with LPS.

Table II. Basic patient information ( $\mathrm{n}=20$ in each group).

\begin{tabular}{lcc}
\hline Parameter & IDD patients & $\begin{array}{c}\text { Spinal cord } \\
\text { injury (control) }\end{array}$ \\
\hline Mean age (years) & $54.9 \pm 7.5$ & $42.2 \pm 8.9$ \\
Gender & $12 \mathrm{M}, 8 \mathrm{~F}$ & $13 \mathrm{M}, 7 \mathrm{~F}$ \\
Segment & L3-L5 & L2-L5 \\
MRI grade & IV & I
\end{tabular}

IDD, intervertebral disc degeneration patients in the experimental group; MRI, magnetic resonance imaging; F, female; $\mathrm{M}$, male.

subjects $(\mathrm{P}<0.05)$. Simultaneously, the miR-27a expression level in NP cells after stimulation with LPS was evidently increased compared with the untreated control cells $(\mathrm{P}<0.05)$. These data indicated that miR-27a was upregulated in IDD patients, as well as in LPS-stimulated NP cells, suggesting that miR-27a may be involved in the development of IDD.

Downregulation of miR-27a decreases proinflammatory cytokine levels in LPS-stimulated NP cells. In order to investigate the role of miR-27a in IDD, a stable NP cell line exhibiting reduced miR-27a expression was generated by transfection of NP cells with an miR-27a inhibitor, while negative control oligonucleotides was used as the negative control group. Subsequently, the cells were stimulated with LPS. At $24 \mathrm{~h}$ after the stimulation, miR-27a expression was analyzed by RT-qPCR, while the mRNA and protein levels of proinflammatory cytokines were detected by RT-qPCR and ELISA, respectively. The results suggested that, following transfection with the miR-27a inhibitor, miR-27a expression was efficiently downregulated (Fig. 2A; $\mathrm{P}<0.05$ ). Compared with the LPS-stimulated only group, the downregulation of miR-27a resulted in a significant decrease in the mRNA (Fig. 2B-D) and protein (Fig. 3) expression levels of proinflammatory cytokines IL-1 $\beta$, IL- 6 and TNF- $\alpha(\mathrm{P}<0.05)$. By contrast, negative control oligonucleotides presented no significant effect on the production of IL-1 $\beta$, TNF- $\alpha$ and IL-6 (Figs. 2 and 3). The data indicated that downregulation of miR-27a significantly decreased the proinflammatory cytokine expression in LPS-stimulated NP cells.

Downregulation of miR-27a suppresses the activation of p38/mitogen activated protein kinases (MAPK) in $L P S$-stimulated NP cells. The present study further investigated whether miR-27a was able to regulate inflammation via the MAPK signaling pathway using western blot analysis. Following stimulation with LPS for $24 \mathrm{~h}, \mathrm{p} 38$ phosphorylation and NF- $\mathrm{\kappa B}$ protein expression were detected using western blot analysis (Fig. 4A). As presented in Fig. 4B and C, downregulation of the miR-27a expression in LPS-stimulated NP cells significantly reduced the protein expression levels of p-p38 and NF- $\kappa B$ when compared with the LPS-simulated NP cells without miR-27a inhibitor $(\mathrm{P}<0.05)$. However, negative control oligonucleotides exhibited no significant effect on the expression levels of p-p38 and NF- $\mathrm{kB}$ in LPS-stimulated NP cells. In addition, RT-qPCR was further performed to 

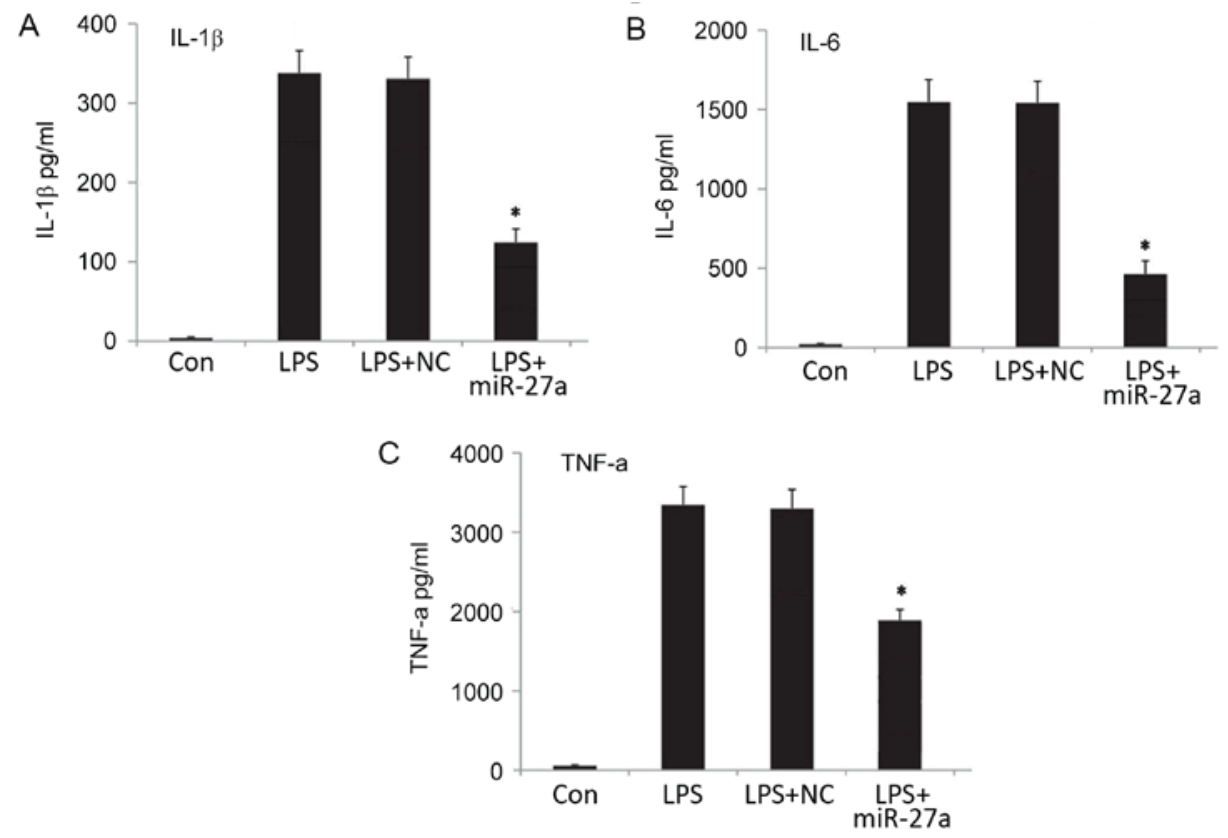

Figure 3. Expression levels of proinflammatory factors (A) IL-1 $\beta$, (B) IL-6 and (C) TNF- $\alpha$ in the cellular supernatant, as determined by ELISA. "P $<0.05$ vs. Con group. All results presented as the mean \pm standard deviation of three independent experiments. miR, microRNA; LPS, lipopolysaccharide; Con, control; NC, negative control vector; IL, interleukin; TNF, tumor necrosis factor; Con, control group, cells without any treatment; LPS group, cells treated with LPS; NC+LPS, cells transfected with negative control and then treated with LPS; LPS+miR-27a, cells transfected with miR-27a inhibitor and then treated with LPS

A

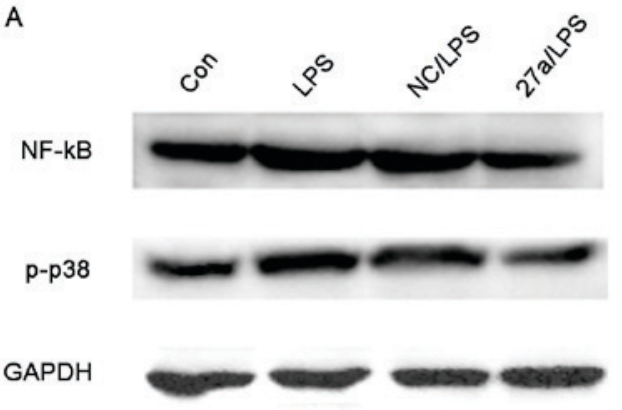

C

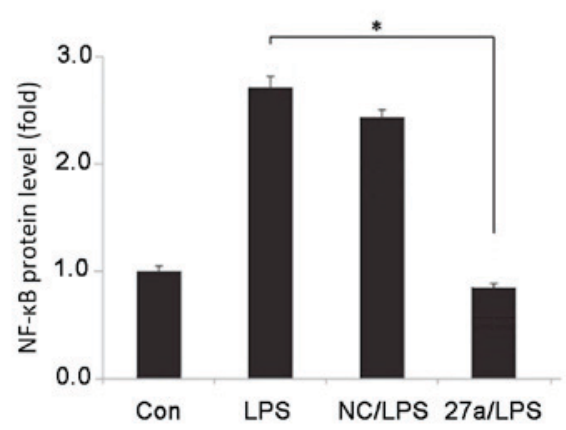

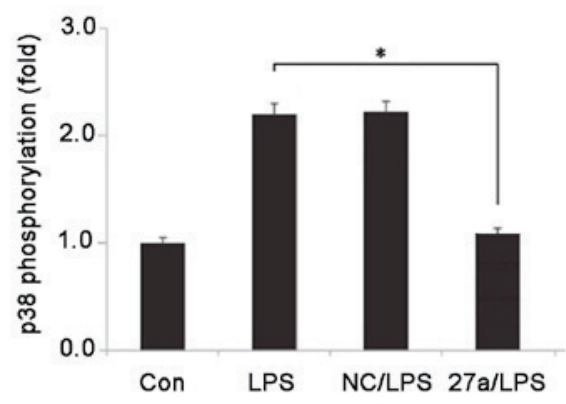

D

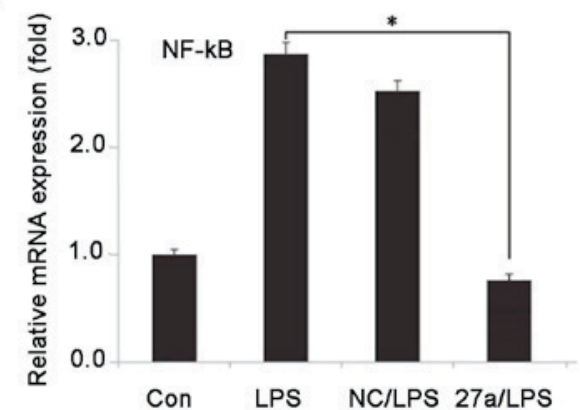

Figure 4. Effect of miR-27a on p38/MAPK pathway in LPS-stimulated human nucleus pulposus cells. (A) Western blots of NF-kB and p-p38 protein expression

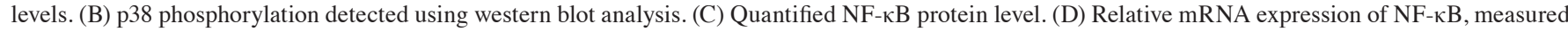
using reverse transcription-quantitative polymerase chain reaction. GAPDH was used as an internal control. ${ }^{*} \mathrm{P}<0.05$ vs. Con group. All results presented as the mean \pm standard deviation of three independent experiments. miR, microRNA; LPS, lipopolysaccharide; Con, control; NC, negative control vector; NF, nuclear factor; MAPK, mitogen-activated protein kinase; Con, control group, cells without any treatment; LPS group, cells treated with LPS; NC+LPS, cells transfected with negative control and then treated with LPS; LPS+miR-27a, cells transfected with miR-27a inhibitor and then treated with LPS.

measure the mRNA expression level of $N F-\kappa B$, which is presented in Fig. 4D $(\mathrm{P}<0.05)$. Changes in the NF- $\kappa \mathrm{B}$ mRNA level were consistent with those in the protein levels. These results suggested that downregulation of miR-27a was able to 
suppress the activation of the p38/MAPK in LPS-stimulated NP cells. All these data suggest that miR-27a may function as a promoter in IDD development, and that inhibition of miR-27a may ameliorate inflammation via the p38/MAPK-signaling pathway in IVD cells.

\section{Discussion}

Increasing evidence has indicated that miRNAs serve critical roles in a number of normal biological and pathological processes, including embryogenesis, lineage determination, as well as in the regulation of cell differentiation, proliferation and apoptosis (26). However, knowledge of the aberrant expression and roles of miRNAs in IDD remain largely uncharacterized. Therefore, identification of IDD-associated miRNAs and exploration of their roles in IDD may be important for developing novel targets for IDD therapy. In the present study, the role of miR-27a in IDD, as well as the pathological links between miR-27a, IDD and inflammatory pathways associated with IDD, were investigated.

Studies have been suggested that an abnormal expression of miR-27a was associated with various diseases, and that the expression of miR-27a was upregulated in degenerative NP cells (19,27-29). However, the role of miR-27a in IDD remains to be investigated. In present study, the expression level of miR-27a in IDD was first verified using RT-qPCR, and the results confirmed that upregulation of miR-27a was observed in IDD.

Inflammation serves a critical role in disc degeneration. Endogenous factors, including crystal deposits in the annulus of human intervertebral discs and ECM breakdown products, can trigger the IVD inflammatory response $(30,31)$. Various proinflammatory cytokines, including IL-1 $\beta$, IL-6, IL-12 and TNF- $\alpha$, were significantly increased due to immunoreactivity in herniated and generative IVD tissues $(32,33)$. To investigate the role of miR-27a in IDD, a stable miR-27a-knockdown NP cell line was generated by transfection with an miR-27a inhibitor, and an IDD cell model was established by LPS stimulation of cells. RT-qPCR and ELISA assay were then performed for the detection of proinflammatory cytokine expression levels in the cells. The results demonstrated that downregulation of miR-27a significantly reduced the levels of proinflammatory cytokines, including IL-1 $\beta$, TNF- $\alpha$ and IL- 6 . This indicated that inhibition of miR-27a was able to suppress inflammatory response in IVD cells.

MAPK signaling pathways are responsible for regulating a variety of cellular activities, including proliferation, differentiation, and apoptosis, in response to certain environmental stimuli. Three major MAPK pathways have been identified in mammals: MAPK/ERK, SAPK/JNK and p38 MAPK. p38 MAPK is activated by external stress, inflammatorycytokines or UV radiation. A previous study reported that the p38 MAPK pathway is involved in development of IDD (34). Therefore, the present study aimed to further investigate the underlying mechanism of the regulation of cell immunoreactivity by miR-27a and the p38/MAPK pathway was also assessed in the current study. As a result of miR-27a downregulation, the p38 phosphorylation was notably decreased. Furthermore, the mRNA and protein expression levels of $\mathrm{NF}-\kappa \mathrm{B}$ were significantly reduced in the LPS-stimulated NP cells. These results suggested that downregulation of miR-27a was able to suppress the activation of the p38/MAPK pathway in LPS-stimulated NP cells.

In conclusion, the present study revealed that miR-27a may function as a promoter in the development of IDD. Inhibition of miR-27a may suppress inflammatory factors released by IVD cells by regulating the MAPK signaling pathway. To the best of our knowledge, this is the first study addressing the underlying mechanisms of miR-27a in IDD, and these findings also highlight miR-27a as a novel potential therapeutic target for IDD.

\section{References}

1. Vos T, Flaxman AD, Naghavi M, Lozano R, Michaud C, Ezzati M, Shibuya K, Salomon JA, Abdalla S, Aboyans V, et al: Years lived with disability (YLDs) for 1160 sequelae of 289 diseases and injuries 1990-2010: A systematic analysis for the Global Burden of Disease Study 2010. Lancet 380: 2163-2196, 2012.

2. Gore M, Sadosky A, Stacey BR, Tai KS and Leslie D: The burden of chronic low back pain: Clinical comorbidities, treatment patterns, and health care costs in usual care settings. Spine (Phila Pa 1976) 37: E668-E677, 2012.

3. Millecamps M, Tajerian M, Naso L, Sage EH and Stone LS: Lumbar intervertebral disc degeneration associated with axial and radiating low back pain in ageing SPARC-null mice. Pain 153: 1167-1179, 2012.

4. Adams MA and Roughley PJ: What is intervertebral disc degeneration, and what causes it? Spine (Phila Pa 1976) 31: 2151-2161, 2006.

5. Mayer JE, Iatridis JC, Chan D, Qureshi SA, Gottesman O and Hecht AC: Genetic polymorphisms associated with intervertebral disc degeneration. Spine J 13: 299-317, 2013.

6. Freemont AJ: The cellular pathobiology of the degenerate intervertebral disc and discogenic back pain. Rheumatology (Oxford) 48: 5-10, 2009

7. Li Z, Liang J, Wu WK, Yu X, Yu J, Weng X and Shen J: Leptin activates RhoA/ROCK pathway to induce cytoskeleton remodeling in nucleus pulposus cells. Int J Mol Sci 15: 1176-1188, 2014.

8. Li Z, Shen J, Wu WK, Yu X, Liang J, Qiu G and Liu J: The role of leptin on the organization and expression of cytoskeleton elements in nucleus pulposus cells. J Orthop Res 31: 847-857, 2013.

9. Ellman MB, Kim JS, An HS, Chen D, KC R, An J, Dittakavi T, van Wijnen AJ, Cs-Szabo G, Li X, et al: Toll-like receptor adaptor signaling molecule MyD88 on intervertebral disk homeostasis: In vitro, ex vivo studies. Gene 505: 283-290, 2012.

10. Iwata M, Ochi H, Asou Y, Haro H, Aikawa T, Harada Y, Nezu Y, Yogo T, Tagawa M and Hara Y: Variations in gene and protein expression in canine chondrodystrophic nucleus pulposus cells following long-term three-dimensional culture. PLoS One 8: e63120, 2013

11. Lee RC, Feinbaum RL and Ambros V: The C. Elegans heterochronic gene lin-4 encodes small RNAs with antisense complementarity to lin-14. Cell 75: 843-854, 1993.

12. Bartel DP: MicroRNAs: Genomics, biogenesis, mechanism, and function. Cell 116: 281-297, 2004.

13. Teague EM, Print CG and Hull ML: The role of microRNAs in endometriosis and associated reproductive conditions. Hum Reprod Update 16: 142-165, 2010.

14. Croce CM: Causes and consequences of microRNA dysregulation in cancer. Nat Rev Genet 10: 704-714, 2009.

15. Wang HQ, Yu XD, Liu ZH, Cheng X, Samartzis D, Jia LT, Wu SX, Huang J, Chen J and Luo ZJ: Deregulated miR-155 promotes Fas-mediated apoptosis in human intervertebral disc degeneration by targeting FADD and caspase-3. J Pathol 225: 232-242, 2011.

16. Yu X, Li Z, Shen J, Wu WK, Liang J, Weng X and Qiu G: MicroRNA-10b promotes nucleus pulposus cell proliferation through RhoC-Akt pathway by targeting HOXD10 in intervetebral disc degeneration. PLoS One 8: e83080, 2013.

17. Ohrt-Nissen S, Dessing KB, Rossing M, Lajer C, Vikeså J, Nielsen FC, Friis-Hansen L and Dahl B: Characterization of miRNA expression in human degenerative lumbar disks. Connect Tissue Res 54: 197-203, 2013.

18. Liu H, Huang X, Liu X, Xiao S, Zhang Y, Xiang T, Shen X, Wang $G$ and Sheng B: miR-21 promotes human nucleus pulposus cell proliferation through PTEN/AKT signaling. Int J Mol Sci 15: 4007-4018, 2014. 
19. Liu G, Cao P, Chen H, Yuan W, Wang J and Tang X: MiR-27a regulates apoptosis in nucleus pulposus cells by targeting PI3K. PLoS One 8: e75251, 2013.

20. Ji J,Zhang J, Huang G, Qian J, Wang X and Mei S: Over-expressed microRNA-27a and $27 \mathrm{~b}$ influence fat accumulation and cell proliferation during rat hepatic stellate cell activation. FEBS Lett 583: 759-766, 2009.

21. Liu T, Tang H, Lang Y, Liu M and Li X: MicroRNA-27a functions as an oncogene in gastric adenocarcinoma by targeting prohibitin. Cancer Lett 273: 233-242, 2009.

22. Ma Y, Yu S, Zhao W, Lu Z and Chen J: miR-27a regulates the growth, colony formation and migration of pancreatic cancer cells by targeting Sprouty2. Cancer Lett 298: 150-158, 2010.

23. Zhao B, Yu Q, Li H, Guo X and He X: Characterization of microRNA expression profiles in patients with intervertebral disc degeneration. Int J Mol Med 33: 43-50, 2014.

24. Pfirrmann CW, Metzdorf A, Zanetti M, Hodler J and Boos N: Magnetic resonance classification of lumbar intervertebral disc degeneration. Spine (Phila Pa 1976) 26: 1873-1878, 2001.

25. Zhao L, Lu X and Cao Y: MicroRNA and signal transduction pathways in tumor radiation response. Cell Signal 25: 1625-1634, 2013.

26. Ell B and Kang Y: MicroRNAs as regulators of bone homeostasis and bone metastasis. Bonekey Rep 3: 549, 2014.

27. Zhao X, Yang L and Hu J: Down-regulation of miR-27a might inhibit proliferation and drug resistance of gastric cancer cells. J Exp Clin Cancer Res 30: 55, 2011.

28. Drayton RM, Dudziec E, Peter S, Bertz S, Hartmann A, Bryant HE and Catto JW: Reduced expression of miRNA-27a modulates cisplatin resistance in bladder cancer by targeting the cystine/glutamate exchanger SLC7A11. Clin Cancer Res 20: 1990-2000, 2014.
29. Hezova R, Kovarikova A, Bienertova-Vasku J, Sachlova M, Redova M, Vasku A, Svoboda M, Radova L, Kiss I, Vyzula R and Slaby O: Evaluation of SNPs in miR-196-a2, miR-27a and miR-146a as risk factors of colorectal cancer. World J Gastroenterol 18: 2827-2831, 2012.

30. Gruber HE, Norton HJ, Sun Y and Hanley EN Jr: Crystal deposits in the human intervertebral disc: Implications for disc degeneration. Spine J 7: 444-450, 2007.

31. Wuertz K, Vo N, Kletsas D and Boos N: Inflammatory and catabolic signalling in intervertebral discs: The roles of NF-kB and MAP kinases. Eur Cell Mater 23: 103-120, 2012.

32. Kokubo Y, Uchida K, Kobayashi S, Yayama T, Sato R, Nakajima H, Takamura T, Mwaka E, Orwotho N, Bangirana A and Baba H: Herniated and spondylotic intervertebral discs of the human cervical spine: Histological and immunohistological findings in 500 en bloc surgical samples. Laboratory investigation. J Neurosurg Spine 9: 285-295, 2008.

33. Shamji MF, Setton LA, Jarvis W, So S, Chen J, Jing L, Bullock R, Isaacs RE, Brown $\mathrm{C}$ and Richardson WJ: Proinflammatory cytokine expression profile in degenerated and herniated human intervertebral disc tissues. Arthritis Rheum 62: 1974-1982, 2010.

34. Liu C, Yang H, Gao F, Li X, An Y, Wang J and Jin A: Resistin promotes intervertebral disc degeneration by upregulation of ADAMTS-5 through p38MAPK signaling pathway. Spine (Phila Pa 1976) 41: 1414-1420, 2016. 